\title{
Le métier de référenceur est-il pérenne et légitime en communication?
}

\author{
Domenget Jean-Claude, Maître de conférences \\ Université de Franche-Comté, ELLIADD-OUN \\ jean-claude.domenget@univ-fcomte.fr \\ Michel Jean-Luc, Professeur des universités \\ Université de Saint-Etienne, \\ CECCOPOP/LARGOTEC \\ jean.luc.michel@mac.com
}




\title{
Résumé
}

Si la pérennité de ce métier de référenceur n'est plus remise en cause, la question centrale qui reste posée est celle de sa légitimité. Un des objectifs de cet article est de montrer que le métier de référenceur s'inscrit dans la structuration générale des professions de la communication. En premier lieu, il s'agira de valider sa pérennité au travers des diversifications et des recompositions des tâches autour d'un noyau invariant de compétences. En second lieu, l'étude analysera le long cheminement vers la légitimité du référencement en montrant l'élargissement de ses tâches opérationnelles du début à des missions de plus en plus stratégiques et globales. Pour cela, une modélisation dynamique est proposée autour de cinq profils type de référenceurs : les Défricheurs, les Explorateurs, les Organisateurs, les Planificateurs et les Stratèges. Cette modélisation vise à offrir une représentation la plus fidèle possible d'une réalité complexe et mouvante tout en s'inscrivant dans le cadre général de la problématique de l'employabilité. Le référencement vise à franchir une nouvelle étape, celle de gagner sa légitimité, c'est-à-dire d'être jugé assez crédible pour participer aux choix stratégiques en amont de la communication par le web.

Mots-clefs : employabilité, légitimation, professionnalisation, référenceur, optimisation

\begin{abstract}
If the sustainability of the SEO is no longer questioned, the central question that remains is that of legitimacy. One objective of this article is to show that the job of SEO is in the general arrangement of the communication professions. First, it validates its sustainability through diversification and reconstructions tasks around an invariant core competencies. Secondly, the study analyzes the long journey to the legitimacy of SEO showing the expansion from its operational early tasks to an increasingly strategic and global role. For this purpose, a dynamic model is proposed based on five types of profiles SEOs : the Pioneers, Explorers, Organizers, Planners and Strategists. This model aims to provide the most accurate possible representation of a complex and changing reality while in the wider context of the issue of employability. SEO aims to go one step further, the gain legitimacy, that is to say to be considered credible enough to participate in the strategic choices upstream communication via the web.
\end{abstract}

Keywords: SEO, professionalization, communication professions, employability, legitimacy 
Il y a une quinzaine d'années, au milieu / à la fin des années 90 pour les plus anciens, des pionniers du référencement ont pressenti l'importance des moteurs de recherche, dont les usages sont aujourd'hui banalisés (Simonot, 2008 ; Simonot et Gallezot, 2009). Autodidactes (Bezille-Lesquoy, 2003), ces pionniers vont s'autoformer en partageant leurs savoirs, savoir-faire, savoir-être issus de tests, d'échanges et d'expériences. Cette autoformation est le seul gage, à titre individuel comme collectif, du maintien d'une employabilité, ce « capital personnel que chacun doit gérer et qui est constitué de la somme de ses compétences mobilisables » (Boltanski et Thévenot, 1991, p. 156). Après une quinzaine d'années de professionnalisation (Dubar, Tripier et Boussard, 2011), si la pérennité du référencement n'est plus remise en cause, à l'image d'autres métiers de la communication, la question centrale qui reste posée est celle de sa légitimité et de son inscription dans les cercles de décision stratégique des entreprises ou des organisations (Michel, 2008).

Un des objectifs de cet article est de montrer que le métier de référenceur s'inscrit dans la structuration générale des professions de la communication. En premier lieu, il s'agira de valider sa pérennité au travers des diversifications et des recompositions des tâches autour d'un noyau invariant de compétences. En second lieu, l'étude analysera le long cheminement vers la légitimité du référencement en montrant l'élargissement de ses tâches opérationnelles du début à des missions de plus en plus stratégiques et globales.

\section{Cadre théorique : autoformation, employabilité et pérennité du métier}

\subsection{Une autre génération d'autodidactes}

«Les penseurs de l'autoformation ont, depuis longtemps, souligné l'aspect social des apprentissages par soi-même » (Cyrot, 2012). La dimension collective dans ces formes d'apprentissage, les formes de sociabilité soutenant l'autodidaxie ont été au cœur de nombreuses recherches (Bezille-Lesquoy, 2003). Aujourd'hui l'augmentation exponentielle des sources d'information et des opportunités relationnelles offertes par les médias sociaux offre «une perspective forte et fascinante pour repenser l'apprentissage, le savoir et la connexion avec les autres » (Thompson, 2010, cité dans Cyrot, 2012).

En nous intéressant aux référenceurs pionniers, nous nous rapprochons moins de ces nouveaux types d'«autoformants» que des autodidactes, ayant appris l'informatique dans le monde du travail au cours des années 1980. Bonvin et Faquier (2010) ont analysé comment l'apprentissage à l'âge adulte de l'informatique a été l'occasion pour une génération d'autodidactes de faire valoir une compétence réelle, issue de caractéristiques biographiques, et de la transformer en qualité 
professionnelle, laquelle n'avait pas trouvé à être employée ou était restée invisible. Dans les deux cas, il s'agissait d'apprendre une discipline qui n'existait pas auparavant, en s'appuyant sur un apprentissage collectif par observations ou échanges d'informations, de savoir-faire, de services. Une prise de conscience rapide des enjeux d'une telle formation leur a permis de faire preuve «d'une volonté curieuse d'apprentissage » (Archambault et Vieille, 1998, cité dans ibid., p. 77-78). De nombreuses qualités, socialement construites mais non encore reconnues, ont permis à ces usagers de faire valoir leurs compétences (dispositions à l'autodidaxie, disponibilité, curiosité, goûts pour la technique, persévérance).

L'autoformation est le seul gage, à titre individuel comme collectif, du maintien d'une employabilité, perçue à la fois comme l'aptitude des individus à rester employables mais aussi l'évaluation des compétences utiles pour un métier, en fonction des autres individus exerçant le même métier (Othmane, 2011). Pour les référenceurs pionniers, si l'évangélisation du marché a participé à la pérennité du métier, ils ont dû évaluer leurs «compétences mobilisables» (Boltanski et Thévenot, 1991) en anticipant les évolutions du marché, en se situant vis-à-vis de leurs pairs et des autres métiers de la communication.

\subsection{Un métier de la communication pérenne}

Si le métier de référenceur s'intègre dans les professions de la communication, il doit en présenter les principales caractéristiques, aux premiers rangs desquelles (Michel, 2008) :

- Une composition modulaire et dynamique des tâches et des fonctions ;

- Des niveaux de compétences clairement repérés ;

- Des réseaux professionnels reconnus ;

- Une évolution notable vers la stratégie et l'approche globale ;

C'est grâce à celles-ci qu'il développe son employabilité à long terme ainsi que sa crédibilité et sa légitimité.

L'hyperspécialisation d'un côté, la polyvalence de l'autre, le mouvement de segmentation des tâches, des fonctions et leur regroupement dynamique, l'augmentation de la complexité des actions, le resserrement des trajectoires professionnelles mais aussi la mobilité professionnelle imposée par les évolutions du marché forment autant d'éléments typiques de cette phase de pérennisation.

A l'image de la démarche constructiviste adoptée par Boltanski (1992), Boltanski et Thévenot (1991) concernant les cadres, l'analyse des trajectoires professionnelles des référenceurs pionniers permet de saisir le processus de structuration au cours duquel des acteurs professionnels se forment, « en groupe explicite, doté d'un nom, d'organisations, de porte-parole, de systèmes de représentations et de valeurs » (Boltanski, 1992, p. 51). Des groupes, identifiés notamment à des forums (forums de 
WebRankInfo - WRI, Taggle, WebmasterHub, Seosphere pour les plus connus) ${ }^{105}$ ou à l'association SEO Camp ${ }^{106}$ (SEO pour search engine optimization) au niveau de la profession, participent à la construction d'une identité professionnelle (Sainsaulieu,1988; Dubar, 2010), en permettant aux membres d'intégrer des pratiques, des codes, des conventions, des connaissances, des aptitudes, des compétences, des savoir-faire voire des comportements, des cultures propres aux métiers de référenceur.

\section{Cadre méthodologique : interviewer les référenceurs pionniers}

La méthodologie employée repose sur une série d'entretiens concernant les trajectoires professionnelles et les pratiques de formation de référenceurs pionniers parmi les plus connus en France. Nous avons adopté une méthode de recrutement par contact direct, l'un des auteurs étant membre de l'association des référenceurs SEO Camp. Un tiers de la population a été recrutée de proche en proche, afin d'atteindre des référenceurs qui ont changé de métier. Pour construire la population d'étude, le premier critère choisi a été l'antériorité dans le domaine, en visant les référenceurs qui se sont lancés dès le milieu des années 90. Il s'est avéré qu'ils se comptaient sur les doigts d'une main. Ce critère trop restrictif ne permettait pas d'intégrer les référenceurs qui organisent, structurent la communauté des référenceurs aujourd'hui, ni des $"$ francs-tireurs $»{ }^{107}$, plus connus sous le nom de Black Hats ${ }^{108}$. En prenant en compte un second critère, celui de l'expérience, nous avons interviewé des référenceurs qui ont entre 5 et 17 ans d'expérience dans le domaine. Ce sont tous, plus ou moins, des autodidactes. Certains n'ont travaillé que dans le référencement mais beaucoup ont connu une vie professionnelle précédente et d'autres prouvent qu' «il y a une vie après le référencement ». La plupart sont Français et ont réalisé leur carrière en France. Nous avons interviewé également un Québécois, un Belge, un Britannique et l'un des interviewés est expatrié à Vancouver.

Ces référenceurs sont presque tous reconnus, cités, visibles dans la profession. Notre population d'étude ne contient ni acteurs travaillant pour les annuaires et moteurs de recherche leaders de l'époque (Altavista, Yahoo, Voila); ni des salariés

\footnotetext{
${ }^{105}$ Les premiers forums datent de 2002.

${ }^{106}$ http://www.seo-camp.org/ L'association SEO Camp date de 2007.

${ }^{107}$ Dans la suite de l'article, les propos des interviewés sont présentés entre guillemets et en italique.

${ }^{108}$ Le sens du terme black hat en SEO diffère de celui en informatique. Il est employé pour « décrire le comportement d'un référenceur qui utilise des techniques contraires aux guidelines de Google ». http://fr.wikipedia.org/wiki/Black_hat
} 
de Google ou anciens googlers. Pour ces derniers, échanger avec eux sur la stratégie de Google aurait certainement été passionnant mais connaissent-ils réellement la stratégie fine de leur compagnie ? Souhaitent-ils (ou réussiraient-ils) à dépasser le message calibré de la marque ? Ceci nous a semblé relever d'un autre objet d'étude. Notons qu'une seule représentante féminine de la profession a été interviewée.

Vingt-trois entretiens ont été effectués au cours des mois de mars et avril 2013. Réalisés via Skype, ils ont été enregistrés à l'aide d'un outil tiers et ont duré en moyenne $1 \mathrm{~h} 20^{\prime}$. Cinq thématiques ont été abordées concernant la découverte du métier; les représentations des figures de référenceurs; la communauté; les trajectoires professionnelles et les pratiques de formation.

La cohérence de cette population d'étude vient plus des missions effectuées par ces différents professionnels (Michel, 2008) qu'une dénomination de métiers commune. Ils se présentent en effet comme (ou ont occupés des postes de) consultant SEO, chef de projet SEO ou encore référenceur junior / sénior. Au moment de l'étude, parmi les interviewés, 9 sont directeur général ou associé dans une agence SEO ou lancent depuis quelques mois un service qui dépasse le SEO ; 9 sont indépendants ; 2 sont en agence; 2 chez l'annonceur et 1 a une double casquette (indépendant / chez l'annonceur).

Cette profession n'est de toute façon ni « séparée », ni « unifiée », ni « établie », ni «objective» (Dubar, Tripier et Boussard, 2011) et se situe entre l'informatique et la communication; avec une tendance de fond d'un glissement vers cette dernière ${ }^{109}$.

\section{Le métier de référenceur vu par les pionniers}

\subsection{Découverte du référencement, missions, pérennité}

Si l'esprit bon enfant du début, l'absence de cadres pour réaliser les missions, une impression parfois que le métier était «bidon» et surtout la méconnaissance totale du domaine par les clients auraient pu faire craindre à la disparition du métier, il faut plutôt analyser la découverte du référencement dans une optique de promotion de sites personnels. Au tout début, nous sommes en 1996, Google n'existait pas encore, les mots même de référencement ou de référenceur n'avaient pas été inventés, «les outils de recherche étaient très différents ». L'activité consistait à inscrire les sites dans les annuaires leaders qui généraient le plus de trafic comme Dmoz, Yahoo,

\footnotetext{
${ }^{109}$ Nous tenons à remercier chaleureusement (par ordre alphabétique) : Olivier Andrieu - Romain Bellet - Julien Bérard - Sébastien Billard - Laurent Bourrelly - David Cohen - Jean D'Alessandro - David Degrelle - David Durand-Pichard - Jean-François Faure - Renaud Joly - Neil Mc Carthy - Eric Maillart - Taï Phan Thanh - Marie Pourreyron - Kévin Richard - Raphaël Richard - Paul Sanchès - Philippe Sasso - Kaddiern Sauvage - Damien Selosse - Yves Weber - Philippe Yonnet. Leurs propos sont le plus souvent anonymés dans le reste de l'article.
} 
Voila en France, La toile du Québec, Branchez-vous au Québec. Les référenceurs souhaitaient surtout trouver des informations sur le fonctionnement des moteurs (Altavista à l'époque) et « comprendre comment ça marche ».

L'activité principale des référenceurs était d' "évangéliser» un marché « embryonnaire ». "Il y avait une époque où il fallait convaincre les clients de faire $d u$ référencement $»$, explique un interviewé. Les missions du référenceur étaient toutes à inventer : faire l'audit d'un site web, définir une liste de mots clés, remplir des balises. L'objectif était en priorité de faire indexer les sites. Très vite, quelquesuns ont inventé des outils de «crawl» ou des «méta-moteurs » pour améliorer leur positionnement.

Le référencement était «un terrain vierge» avec un "côté magique », quelque chose de «marrant ». «Être en première position » sur une myriade de mots-clefs, augmenter le trafic d'un site («fois 6, fois 10») mais aussi «jouer avec les moteurs » en «les spammant comme des bourrins » avec des pages alias, des pages de contenus et surtout des pages satellites ${ }^{110}$; tout cela créait une vraie effervescence. Les référenceurs arrivés par hasard ou par opportunisme car le Web était nouveau n'avaient souvent pas de vision à long terme de l'activité de référencement, tout en ayant pour certains l'intuition que «le Web allait changer le monde ».

\subsection{Pionniers, autodidactes, experts}

S'ils se reconnaissent dans la très grande majorité comme autodidactes, les référenceurs interviewés sont plus circonspects quant à leur statut de pionniers ou même d'experts.

Deux visions du statut de pionniers ont émergé des entretiens. Les pionniers sont des «défricheurs", les premiers à s'aventurer sur le terrain vierge des outils de recherche. Ils sont donc peu nombreux à être reconnus comme tels (Danny Sullivan aux Etats-Unis et Olivier Andrieu en France notamment). Une autre vision, plus décomplexée, moins révérencieuse envers les anciens a consisté à se considérer «tous comme des pionniers », renvoyant à l'idée qu'il reste possible d'explorer aujourd'hui de nouvelles contrées du référencement, territoires progressivement défrichés par les Black Hats notamment. «L'âge des pionniers » entendu ici dans un sens large est en effet celui des tests.

Si la figure de l'expert peut être considérée comme un modèle du «paysage réputationnel»(Origgi, 2007) des référenceurs qui participe d'un processus de

\footnotetext{
${ }^{110}$ Une page satellite est une page optimisée sur un ou plusieurs mots-clefs, destinée aux moteurs de recherche et visant le plus souvent à contourner un frein au référencement http://www.rankspirit.com/page-satellite.php
} 
singularisation dont le premier enjeu est de se différencier, d'atteindre ce statut d'expert et d'acquérir un nom ; à l'image d'autres métiers de la communication, cette quête d'expertise connaît actuellement un renouveau, à travers sa mise en visibilité sur les médias sociaux. A côté de cette dimension réputationnelle, les interviewés ont dépeint une figure de l'expert marquée surtout par une accumulation d'expériences. «En SEO, ce qui donne de l'expertise, c'est d'avoir travaillé sur des sites différents ${ }^{111}$. En fait, l'expertise est assez différente selon le profil technique ou stratégique du référenceur ainsi que les types d'organisations dans lesquelles ils travaillent (indépendants, en agence, « in house » chez l'annonceur pour une PME, « in house» dans une grande entreprise). A titre d'exemples, un référenceur en agence va conseiller et accompagner différents types de clients (artisans commerçants, PME, grands comptes) sur différents types de sites (sites vitrines, sites e-commerce spécialisés, sites e-commerce généralistes, présence sur des sites de presse ou comparateurs de prix, services de proximité). Un référenceur indépendant va avoir un profil plus technique, travaillant seul et sera spécialisé sur quelques secteurs d'activité et types de sites. Il gère souvent le référencement de ses propres sites Web pour apporter du trafic et les monétiser. Quant au référenceur chez l'annonceur, il travaille sur un site unique ou une galaxie de sites. Selon le profil de l'annonceur, il va définir ou appliquer une stratégie et un ensemble de recommandations touchant à l'optimisation technique, la rédaction de contenus éditoriaux, la stratégie de liens, l'analyse des résultats et les mises à jour du ou des site(s).

\subsection{Communauté, autoformation, réputation}

Pour combattre une éventuelle fragilité du métier de référenceur, l'autoformation auprès des pairs est depuis le début indispensable. Si l'hétérogénité des formations et des parcours d'autoformation caractérise les référenceurs pionniers, il semble indispensable pour tous de "se considérer en formation permanente» dans une profession en changement constant. La dimension sociale de l'autoformation est d'autant plus importante chez eux qu'il fallait rompre avec un isolement professionnel, qu'ils soient indépendants ou en agence. Très vite la recherche d'informations les a conduits à s'inscrire à des listes de diffusion (la liste referencement sur yahoo groups a été la plus citée) puis est venu l'âge des forums. Ces échanges en ligne ont donné lieu ensuite à la naissance d'événements «In Real

\footnotetext{
${ }^{111}$ En effet, « l'alchimie du SEO » est différente entre les blogs, forums, sites e-commerce spécialisés, sites e-commerce généralistes, site de presse, comparateurs de prix, services de proximité, site de banque et assurance. http://www.renaud-joly.fr/index.php/post/2011/2002-referencement
} 
Life (IRL) » pour renforcer les liens entre référenceurs, partager de bons moments ou recruter des collaborateurs.

De ces initiatives est née une communauté SEO qui est une communauté d'intérêt (Proulx, 2006), dans laquelle se sont développés un sentiment communautaire et des règles d'appartenance participant à la création d'une identité collective. Ce qui a fait lien dès le début, c'est ce besoin d'échanges, de partage des tests afin de monter en compétence individuellement par le collectif. Même si des controverses émaillent aujourd'hui encore la vie de la communauté, des scissions ont marqué son histoire, des groupes fermés ont été créés, des tentatives de rassemblement ont été avortées ; l'atmosphère beaucoup plus pacifiée, d'un avis général, traduit une maturité de la profession et une marche vers la légitimité du métier.

Exemple des controverses intestines et sans s'attarder sur les biais d'un tel exercice, la sortie d'un classement du «meilleur » référenceur de France, proposé par le Journal Du Net (JDN) ${ }^{112}$ en 2013, met en lumière les tactiques d'e-réputation développées par de nombreux référenceurs au sein de la communauté. Que ce soient sur les forums, en tant qu'administrateurs, modérateurs, participants aux concours de référencement; sur les blogs, «influents» tout du moins pour faire réagir la communauté, sur Twitter améliorant les mécanismes de construction d'une eréputation (Domenget, à paraître); de nombreux référenceurs ont développé des tactiques pour «se faire un nom », gagner en visibilité, autre gage pour maintenir leur employabilité.

\subsection{Employabilité, évolutions du marché et trajectoires professionnelles}

L'évangélisation du marché effectuée parmi les pionniers par les Explorateurs et les Organisateurs ${ }^{113}$ a été le socle de l'employabilité de l'ensemble des référenceurs. Au delà de la multiplicité des trajectoires professionnelles; le passage de l'agence chez l'annonceur et vice-et-versa, la relative stabilité chez les indépendants, la possibilité de sortir du marché du référencement pour travailler dans la communication ou le webmarketing traduisent l'employabilité des référenceurs, contrastant avec un marché qui a fortement évolué et a connu déjà deux crises économiques importantes (celle de l'éclatement de la bulle Internet en 2000 / 2001 et celle actuelle à partir de 2007 / 2008). Plus qu'une dépendance aux modifications des algorithmes de Google ou aux derniers filtres apparus (Panda puis Pingouin), l'employabilité et les opportunités de carrière d'un référenceur dépendent beaucoup plus des évolutions du marché à travers les attentes des clients, de la

\footnotetext{
112 http://www.journaldunet.com/solutions/seo-referencement/le-meilleur-seo-francais/

${ }^{113}$ Voir la modélisation des profils type de référenceurs pionniers en point 4.
} 
professionnalisation du secteur et comme nous l'avons vu, de la capacité de chacun à évaluer ses « compétences mobilisables » vis-à-vis de ses pairs et des autres métiers de la communication.

Le marché du référencement a toujours été volatile. «Le problème fondamental reste la rétention des clients ». Qu'ils soient indépendants et jouent principalement sur leur réputation ou une agence qui porte en bannière son client historique; le «turn over » de clients est important. Car à la différence des liens sponsorisés avec lesquels la mesure du retour sur investissement est immédiate, le talon d'Achille du SEO reste l'absence de critères de mesure d'efficacité fiables. Les interviewés ont très bien expliqué la part de feeling, d'expérience, de tests qui persiste dans le métier, même si en fonction des typologies de sites des problématiques récurrentes se font jour. Le référencement reste l'affaire d'une «alchimie » qui entretient une part de magie du métier, difficilement conciliable avec les standards d'efficacité marketing attendus aujourd'hui par le marché.

Dans ce domaine, les opportunités professionnelles restent nombreuses mais sont fortement corrélées à une dimension géographique (Paris et sa région, voire Lille, sans commune mesure avec le reste de la France). Il n'existe évidemment pas une trajectoire professionnelle unique, allant de l'opérationnel «avoir la main dans le cambouis » : coder, produire du contenu, partir à la pêche aux liens jusqu'à définir la stratégie SEO ou même la stratégie de communication de grands comptes. Tous les pionniers ne sont pas devenus des stratèges. Certains veulent absolument rester dans leur " cœur de métier », d'autres ont quitté le SEO et sont partis dans des stratégies transversales. Il est tout autant possible de faire le chemin inverse et de passer d'une fonction stratégique à un retour à l'opérationnel. Au delà de ces constats, l'enjeu est de proposer un modèle qui rend compte de la complexité dynamique de ces trajectoires professionnelles.

\subsection{Métiers du référencement, formation, nouveaux profils}

La formation a toujours été au cœur du métier de référenceur. Né de l'autoformation, ce métier a gagné en crédibilité en intégrant dès le départ parmi ses missions la formation des différents publics auxquels il s'adresse. Parmi ces publics, nous pouvons distinguer les clients, les collaborateurs, les dirigeants, les pairs et les étudiants :

- du côté des clients, même si nous ne sommes plus à l'âge de l'évangélisation du marché ${ }^{114}$, les consultants SEO continuent à former les clients, dans le sens de leur faire prendre conscience des nouveaux enjeux du référencement ;

\footnotetext{
${ }^{114}$ Les explorateurs qui se lancent dans de nouveaux services e-marketing redécouvrent cette phase d'évangélisation du marché.
} 
- du côté des collaborateurs, la formation consiste à éduquer les autres professionnels du Web (webdesigner, développeur, rédacteur) au respect des fondamentaux du référencement et aux bonnes pratiques SEO ;

- du côté des dirigeants, les stratèges ont dans leurs missions un rôle beaucoup plus «politique »;

- du côté des pairs, l'autoformation est passée aujourd'hui des forums à Twitter, devenu un canal de veille prédominant dans le secteur mais aussi en interne au sein d'équipe SEO, lors d'événements IRL ou même auprès de Blacks Hats qui ont lancé une formation sur les usages d'outils avancés de recherche et de création de liens ;

- du côté étudiant, les formations initiales et/ou en alternance en référencement sont récentes $(2008$ / 2009) et restent rares (citons principalement la licence professionnelle de rédacteur référenceur web à Mulhouse et la licence professionnelle de marketing digital à Lyon). Au delà d'une controverse chez les référenceurs pionniers, liée à la croyance de certains que seule la reproduction de leurs parcours d'autodidactes permet de se former à ces métiers, l'arrivée de référenceurs diplômés dans le domaine participe clairement d'une légitimation de la profession.

Cette arrivée conduit également à s'interroger sur l'évolution des profils de référenceur. Une tendance de la professionnalisation et de la quête de légitimité semble distinguer d'un côté des référenceurs opérationnels hyperspécialisés, se rapprochant d'un profil technique (avec une spécialisation par exemple sur l'architecture des sites ou un $\mathrm{CMS}^{115}$ notamment d'e-commerce) et à l'opposé des stratèges, se rapprochant d'un profil communication, dépassant le cœur de métier SEO pour réfléchir à la place du SEO parmi l'ensemble des leviers e-marketing dans une stratégie de visibilité sur le Web ou le mobile. Entre les deux, un ensemble de profils plus ou moins polyvalents perdure.

\section{Modélisation des profils type d'acteurs et des formations}

De l'étude menée auprès de vingt-trois référenceurs pionniers, il ressort qu'il est possible de les regrouper en cinq grands profils types. Ces profils ne sont pas exclusifs les uns des autres. Un référenceur peut ainsi entrer dans le métier avec un profil et le conserver tout au long de son activité tandis qu'un autre pourra passer graduellement de l'un à l'autre en parcourant plusieurs niveaux de compétences.

\footnotetext{
${ }^{115}$ Un CMS ou Content Management System est un type de logiciels destinés à la conception et à la mise à jour dynamique de sites Web ou d'application multimédia http://fr.wikipedia.org/wiki/Syst\%C3\%A8me_de_gestion_de_contenu
} 
La modélisation proposée dans cet article se veut dynamique. Cette méthode caractérise des types par des appellations visualisables et génériques. Elle permet d'offrir une représentation la plus fidèle possible d'une réalité complexe et mouvante, tout en s'inscrivant dans le cadre général de la problématique de l'employabilité d'un nouveau métier en butte permanente aux interrogations sur sa pérennité.

Les cinq profils (voir Figure 6) type ont émergé des entretiens et de leurs recoupements. Rappelons qu'il s'agit d'une méthode qui permet d'offrir une représentation à la fois souple, dynamique et précise d'une réalité complexe sans pour autant la réduire à une cybernétique comportementale réductrice.

Une fois ces profils construits et mis en perspective, ils seront croisés avec la question des formations montées ou fréquentées par les pionniers, dans le triple but de se tenir au courant des évolutions, d'échanger entre pairs et d'augmenter leur crédibilité professionnelle.

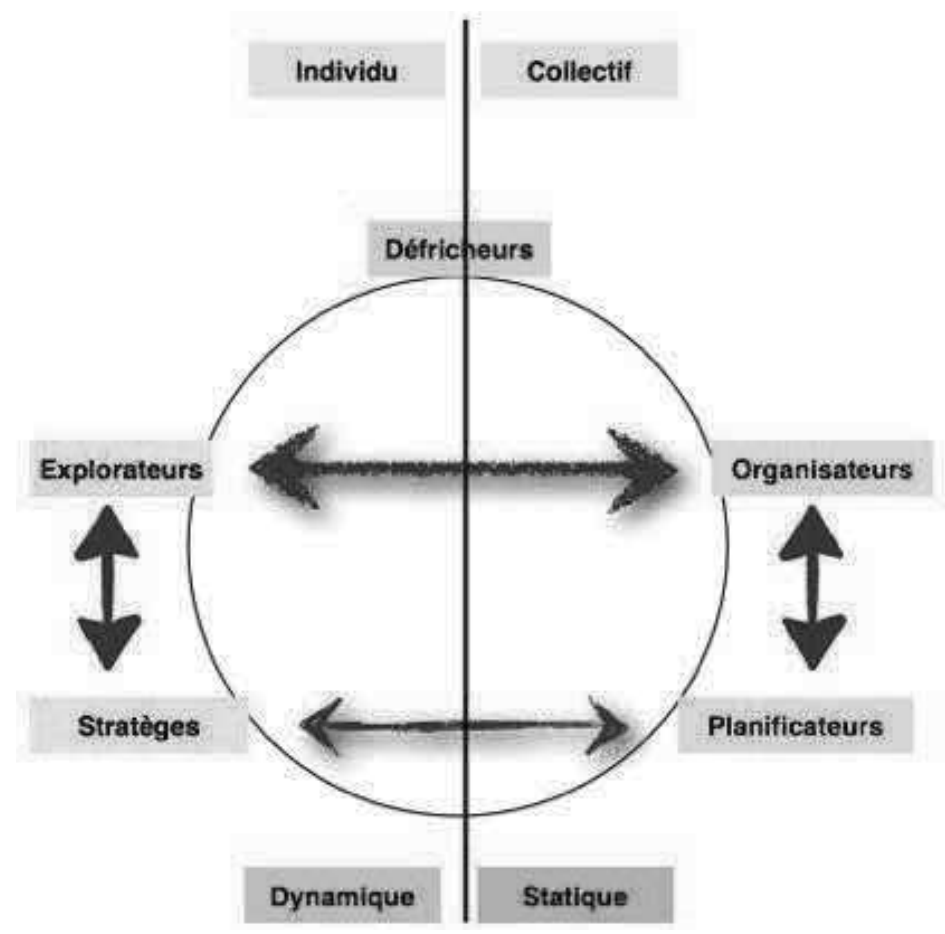

FIGURE 7

Modélisation des 5 profils type de référenceurs pionniers 
Le schéma ci-dessus présente les cinq profils types en les regroupant par grandes unités.

Les Défricheurs sont à la source de la profession de référenceurs. Ils ont engendré les Explorateurs et les Organisateurs. Ceux-ci donnant naissance à leur tour aux Stratèges et aux Planificateurs. Sur la gauche se situe l'axe de l'individu et du dynamisme et à droite l'axe du statique et du collectif. Cette question individu / collectif apparaît différenciante pour ne pas dire clivante entre eux. L'axe à gauche correspond aux profils (Explorateurs et Stratèges) qui sont dans l'évolution des métiers. Tandis que l'axe à droite intègre les profils (Organisateurs et Planificateurs) qui consolident la profession. Sans être dans un désir d'institutionnalisation, ils savent que la légitimité passe par l'organisation. Voyons à présent en détail ces cinq profils type.

\section{Profil 1. Les Défricheurs}

Dans la population étudiée, mais plus largement aussi, ils sont très rares, de l'ordre de quelques personnes en France. Ce sont eux qui ont construit l'histoire du métier de référencement dans une totale improvisation face à de nouvelles pratiques de communication. Ils ont défini les premières missions, se sont posés les premières questions. Ils venaient d'univers très distincts, de l'informatique, de la communication mais aussi d'autres disciplines sans rapport avec le net. Ils ont essuyé les premières difficultés et ont posé les premières problématiques de la recherche pertinente sur le web. La quasi totalité (au moins de la population étudiée) ne s'est pas arrêtée en route et a poursuivi vers les autres profils. Comme dans d'autres secteurs de l'informatique, par exemple la réflexion sur les interfaces homme / machine, on leur doit les premiers essais de facilitation des accès aux sources dans un univers où les sites étaient encore très peu nombreux.

\section{Profil 2. Les Explorateurs}

Présentant le même esprit indépendant que les Défricheurs, ils se sont attaqués à la diversité des premiers moteurs, du genre Lycos, Altavista et autres. Les informations étaient rares mais très qualitatives, ce qui leur a permis de mettre au point des procédures personnelles souvent performantes. Les Explorateurs ont le désir de se dépasser, ils ne se préoccupent guère de créer des structures durables, ils sont dans l'opérationnel, souvent dans le code, ils recherchent la performance maximale. Ils n'ont ni crainte, ni obsession de la concurrence, ils comptent avant tout sur leurs propres ressources. 
Lorsqu'ils communiquent sur leur activité, c'est essentiellement sur la base du partage, même lorsqu'ils sont devenus célèbres, à l'instar d'un Olivier Andrieu par exemple.

Les Explorateurs croient dans leurs capacités de réussite personnelle. Ils pensent que les systèmes, même les plus perfectionnés, y compris les évolutions imposées par Google laissent toujours une marge de manœuvre, des espaces intersticiels de liberté et d'action. Une partie des Explorateurs a fait ses classes dans les concours de positionnement en tant que «Mangeurs de cigogne ${ }^{116}$. On retrouve aujourd'hui des Explorateurs dans la communauté Black Hats.

\section{Profil 3. Les Organisateurs}

Parmi tous les pionniers rencontrés pour cette étude, ce sont eux qui ont construit les premières missions SEO et la structuration des premiers réseaux, la création des premières agences avec l'intégration d'outils de référencement souvent créés par eux-mêmes.

Ils ont eu conscience, souvent très tôt, qu'une simple mission d'explorateur ne pouvait pas dégager de pérennité ni d'employabilité. C'est pourquoi ils ont cherché à échanger des informations, à s'entraider, à créer et / ou fréquenter les forums et pratiquer une autoformation appuyée sur l'expérience collective et plus tard sur l'intelligence collective au sens de Pierre Lévy.

Plus tard ils ont participé à la création d'associations déclarées ou non, dont les deux premières tentatives (IPEA et SEMA7) se sont soldées par un échec, avant que l'association SEO Camp ne réussisse à dépasser les « coteries historiques » entre ses membres. En parallèle, la montée en charge de Google a rebattu les cartes et imposé de nouveaux changements.

\section{Profil 4. Les Planificateurs}

Face aux attentes des clients, à ce qui était ressenti à tort ou à raison comme l'hégémonie toute-puissante de Google, les planificateurs ont essayé d'appliquer les techniques classiques du management. Essayant de démasquer les faux discours d'expertise et les dérives des agences SEO incompétentes, ils ont progressivement facilité l'intégration des agences de communication qui étaient absentes au départ. Ils ont aussi favorisé l'évolution vers davantage de professionnalisme des agences spécialisées en SEO, en manageant des équipes de plus en plus nombreuses nonobstant les problèmes bureaucratiques classiques engendrés par l'extension de la

\footnotetext{
116 «Mangeurs de cigogne » est le nom du premier concours de positionnement organisé sur le forum Webmaster Hub.
} 
planification (et dont il ne sera pas fait état ici). Leur effort de planification permettait selon eux de gagner en crédibilité et d'avancer dans la voie de l'employabilité.

On peut considérer que leur action est déjà préstratégique car elle reconnaît la diversité des missions et a proposé dès le début de modéliser les structures clientes, de segmenter les offres et de trouver des modèles économique pérennes. Ils cherchent à sortir du one shot, marqué par une tarification trop basée sur la performance, pour aller vers un accompagnement inspiré par les grands cabinets d'audit.

Ce sont les Planificateurs qui ont imaginé et réalisé les premiers cycles de formation complets, aussi bien vis-à-vis de leurs clients, de leurs partenaires, de leurs collègues, et parfois, mais rarement (!) des instances universitaires.

Que ce soit pour répondre aux attentes de leurs clients ou par conviction personnelle, ils ont également exposé les premières règles éthiques avec notamment des Guides de bonne conduite, une charte déontologique, même si cela se situait dans une logique imposée par Google.

\section{Profil 5. Les Stratèges}

Les Stratèges dépassent les techniques du SEO pour sortir du rapport opérationnel du référencement. Ils sortent du cœur du métier et s'occupent de communication au sens le plus large, avec des recommandations vis-à-vis de l'image, de l'identité et des valeurs de leurs entreprises clientes. Ils interviennent le plus tôt possible sur le marketing des sites en ne se contentant plus d'optimiser les recherches par diverses ruses ou procédures.

Avec les Stratèges, le référencement s'oriente vers la visibilité en ligne et hors ligne ainsi que l'e-réputation.

La même démarche de crédibilisation croissante de leur activité les a conduits à engager des actions de formation auprès des dirigeants et des décideurs sur des contenus de plus en plus stratégiques et globaux. Ils cherchent à positionner leur métier au plus haut niveau hiérarchique pour mieux exercer une mission complète de communication.

On peut aussi considérer que leur activité prépare les évolutions possibles du référencement, vis-à-vis du web sémantique et d'une « recherche comportementale » symbolisée aujourd'hui par un service comme Siri d'Apple, en intégrant une dimension «politique » (ou stratégique) à leur action. Ils voient celles-ci comme une nouvelle étape - et pas une révolution - qu'ils s'estiment capables d'intégrer sans souci pour l'employabilité grâce à leur approche résolument globale et à leurs compétences toujours entretenues par une veille efficace. 


\subsection{Modélisation des trajectoires professionnelles et des formations}

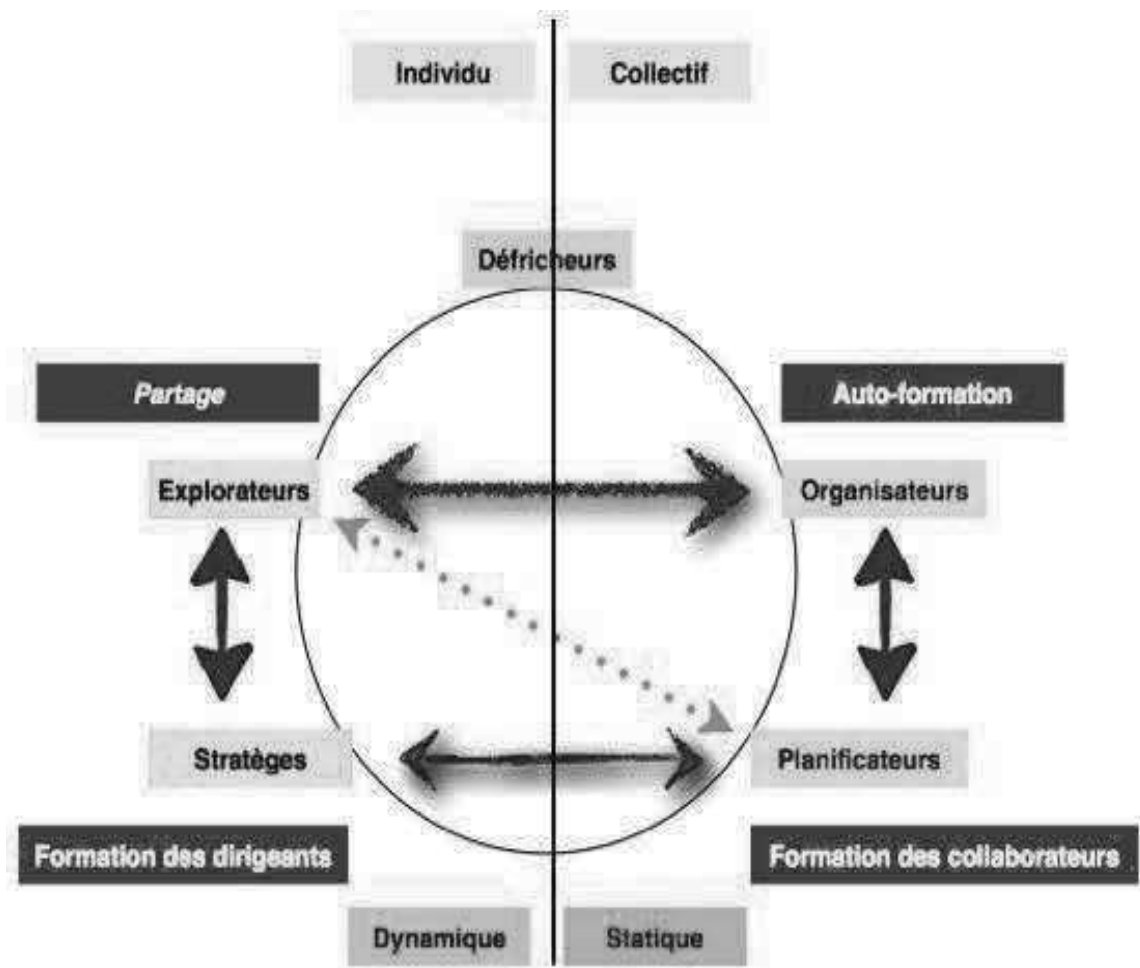

\section{FIGURE 8}

Modélisation des trajectoires professionnelles et des formations chez les référenceurs pionniers

Ce dernier schéma (voir Figure 8) permet d'illustrer la dynamique des trajectoires professionnelles plutôt que de vouloir rechercher des parcours type. Ainsi, certains référenceurs Explorateurs sont restés à ce profil, de même que des Organisateurs ou des Planificateurs. Symétriquement, certains Explorateurs se sont fait Organisateurs via l'autoformation, puis Planificateurs pour accroître la crédibilité de leurs pratiques. Enfin certains se sont fait Stratèges après être passés par les autres profils.

Si l'on superpose les activités de formation sur le schéma, les corrélations avec les profils types apparaissent immédiates :

- Les Explorateurs étaient et restent dans le partage, ils ne tiennent pas à en sortir, leurs témoignages sont gratuits ou peu onéreux ; 
- Les Organisateurs ont créé l'autoformation et ils l'entretiennent toujours via les outils de réseaux : forums, groupes privés, newsletter, association, Twitter, etc. ;

- Les Planificateurs ont monté des programmes de formation segmentés vers leurs clients et leurs partenaires ;

- Quant aux Stratèges, ils en sont déjà à la formation des dirigeants pour renforcer le positionnement stratégique qu'ils souhaitent voir assigné au référencement.

Le schéma illustre aussi certaines des tensions qui peuvent exister entre Explorateurs et Planificateurs, notamment entre le partage et la formation payante des collaborateurs.

On peut aussi considérer que les Explorateurs qui veulent passer à la stratégie ont découvert la nécessité de celle-ci dans les aléas de la planification.

\section{Conclusion : une victoire sur la pérennité et une quête de légitimité}

Cette victoire sur la pérennité est à porter au crédit de tous les référenceurs, bien au delà de leurs différences. Tous cherchaient à inventer un métier réellement nouveau.

La relation des référenceurs avec Google reste ambivalente, à la fois « Google dépendant» et ayant "plus de connaissance sur le fonctionnement du moteur que les équipes en interne ». Paradoxalement, c'est peut-être lui qui a fondé définitivement la pérennité du métier. Certes, les référenceurs étaient prêts : leurs pratiques, leurs astuces, leurs forums, les avaient conduits à un niveau d'expertise et de reconnaissance de leurs compétences leur permettant de se situer favorablement par rapport à leurs clients, mais c'est surtout leur capacité, on pourrait dire à « digérer » toutes les innovations, qui a apporté la preuve de leur durabilité et de leur employabilité, aussi bien en interne (profils assez rares dans la population étudiée) qu'en externe comme consultants. Les profils type découverts dans cette recherche montrent comment ils y sont parvenus, tous avec des recettes différentes, de l'Explorateur au Planificateur.

Beaucoup de nouveaux métiers peuvent se contenter d'avoir acquis leur pérennité et donc leur employabilité durable. Celle-ci débouche le plus souvent sur des programmes de formation ajustés aux besoins et dispensés par les structures privées ou publiques. Le référencement aurait pu s'arrêter là et se développer dans ce cadre, tout en continuant à se diversifier, se recomposer autour de ses invariants qui lui assurent une évolutivité suffisante pour absorber les innovations, petites ou grandes.

S'il s'avère vrai que le référencement s'inscrit bien dans les professions de la communication, une autre étape encore plus difficile doit être franchie : il s'agit de gagner sa légitimité, c'est-à-dire d'être jugé assez crédible pour participer aux choix stratégiques en amont de la communication par le web. Son employabilité atteindra ainsi le niveau du top management, des comités de direction et du consulting. On 
s'en doute, pour une activité, jugée naguère comme une sorte de mal technique nécessaire, simple complément de la création de sites, la route est longue. D'où l'importance du rôle des pionniers dans une évolution aussi considérable. En une quinzaine d'années, grâce aussi bien aux évangélistes, aux experts, à tous les outils de visibilité et de reconnaissance mis en place, grâce aussi à la compétence collective (obérée par certaines agences qui n'y ont vu qu'un marché lucratif), le référencement s'est engagé dans une voie de plus en plus globale, d'abord préstratégique, puis, au fil des succès et de la crédibilité gagnée, de plus en plus stratégique.

La légitimité est-elle pour autant atteinte aujourd'hui ? Les pionniers pensent que oui. Leur connaissance du marché incline à penser qu'ils ont raison et que le métier de référenceur est effectivement devenu stratégique, sans pour autant perdre aucune de ses caractéristiques opérationnelles bien sûr. S'il en est réellement ainsi, le cycle complet aura été accompli. Il n'est pas possible, dans le cadre de cette étude, d'en avoir la certitude, mais la prochaine grande évolution avec le web sémantique permettra d'apporter une nouvelle validation. En recomposant toute la chaîne de production web autour de concepts linguistiques et sémiologiques et à partir de quantifications issues du brain tracking, le web sémantique ouvrira une vaste voie de validation de la percée stratégique et globale du référencement.

\section{Bibliographie}

Aubert, N., et Haroche, C. (dir.) (2011). Les tyrannies de la visibilité. Être visible pour exister?, Toulouse, France : Éditions Érès.

Boltanski, L. (1992). Les cadres. La formation d'un groupe social. Paris, France : Éditions de Minuit.

Bézille-Lesquoy, H. (2003). L'autodidacte. Entre pratiques et représentations sociales. Paris, France : L'Harmattan.

Boltanski, L. et Thévenot, L. (1991). De la justification. Les économies de la grandeur. Paris, France : Gallimard.

Bonvin F., et Faguer, J-P. (2000). Une génération d'autodidactes. Actes de la recherche en sciences sociales,134,76-83. doi : 10.3406/arss.2000.2694

Cyrot, P., et Jeunesse, C. (2012). Autoformation et réseaux virtuels, Distances et médiations des savoirs, (1), repéré à http://dms.revues.org/137

Domenget, J-C. (à paraître). Construire son e-réputation sur Twitter : les pratiques instrumentalisées de professionnels de la visibilité. Dans C. Alcantara (dir.), Eréputation et traces numériques: dimensions instrumentales et enjeux de société. Toulouse, France : Lextenso Éditions. 
Dubar, C. (2010). La crise des identités. L'interprétation d'une mutation (4éd). Paris, France : PUF.

Dubar, C. (1998). La socialisation, Construction des identités sociales et professionnelles ( $4^{\mathrm{e}}$ éd). Paris, France : Armand Colin.

Dubar, C., Tripier P. et Boussard, V. (2011). Sociologie des professions ( $3^{\mathrm{e}}$ éd.). Paris, France : Armand Colin.

Michel, J-L. (2008). Les professions de la communication : fonctions et métiers $\left(3^{\mathrm{e}}\right.$ éd.). Paris, France : Ellipses.

Origgi, G. (2007). Un certain regard. Pour une épistémologie de la réputation. Communication au Workshop « réputation », Fondzione Olivetti, Rome. Repéré à http://cerses.shs.univ-paris5.fr/IMG/pdf/G_Origgi.pdf

Othmane, J. (2011). L'employabilité : définition, création d'une échelle de mesure et contribution à l'étude des déterminants (Thèse de doctorat, université Lyon 3 ). Repéré à http://theses.univlyon3.fr/documents/lyon3/2011/othmane $\mathrm{j} \# \mathrm{p}=3 \mathrm{\&} a=$ top

Proulx, S. (2006). Les communautés virtuelles : ce qui fait lien. Dans S. Proulx, M. Sénécal et L. Poissant (dir.), Communautés virtuelles : penser et agir en réseau (p. 13-25). Québec : Presses Universitaires de Laval.

Sainsaulieu, R. (1988). L'identité au travail. Les effets culturels de l'organisation ( $3^{\mathrm{e}}$ éd.). Paris, France : Presses de Sciences Po.

Simonnot, B. (dir.) (2008). Moteurs de recherche : usages et enjeux. Questions de communication, 14. Repéré à http://questionsdecommunication.revues.org/592

Simonnot, B. \& G. Gallezot (dir.) (2009). L'entonnoir : Google sous la loupe des sciences de l'information et de la communication. Caen, France : C\&F Editions. 